\title{
A survey of anaphylaxis etiology and treatment
}

\author{
Hamid Ahanchian ${ }^{1,2}$, Fatemeh Behmanesh ${ }^{2}$, Farahzad Jabbari Azad ${ }^{3}$, Elham Ansari ${ }^{2}$, Maryam Khoshkhui $^{3}$, Reza Farid $^{3}$, \\ Yalda Hassanpur ${ }^{2,}$, Samaneh Kouzegaran ${ }^{2, *}$ \\ 1 Child Health Research Centre, University of Queensland, Brisbane, Australia \\ 2 Department of Pediatric Allergy and Immunology, School of Medicine, Mashhad University of Medical Sciences, Mashhad, Iran \\ 3 Allergy Research Center, Mashhad University of Medical Sciences, Mashhad, Iran
}

*Correspondence to: Yalda Hasanpur, Asalyalda54@yahoo.com; Samaneh Kouzegaran, kouzegarans@gmail.com. orcid: 0000-0002-2464-4770 (Samaneh Kouzegaran)

\section{Abstract}

Identifying the causes of anaphylaxis which is an acute, potentially fatal systemic reaction is very important in every community. Treatment strategies and pitfalls should also be determined. We sought to determine the most common triggers of anaphylaxis, clinical manifestations and treatment strategies in Mashhad, northeast of Iran. An observational cross-sectional study was conducted to evaluate all patients with a history of anaphylactic reaction who were referred to University Allergy Clinics between 2006 and 2016 in Mashhad Iran. We used a combination of patient's clinical history and allergy diagnostic testing including radioallergosorbant test and skin prick test in order to determine the etiology of anaphylaxis. We identified 172 anaphylactic reactions in 70 patients. Median age was 15 years with a range from 6 months to 48 years. The triggers included: foods, $61.4 \%$; drugs, $15.7 \%$; hymenoptera venom, $8.6 \%$; idiopathic, $5.7 \%$; immunotherapy, $4.3 \%$ and other etiologies: 5.7\%. Nuts and seeds were the most important triggers of food induced anaphylaxis, especially in school children, adolescents and young adults, followed by fruits. However, Cow's milk and hen's egg were the main triggers of anaphylaxis in children aged under 2 years. The most common symptoms were cutaneous and cardiovascular. Corticosteroids $(94.3 \%)$ and/or antihistamines $(85.7 \%)$ were used most frequently for treatment followed by intravenous fluids (54.3\%), whereas epinephrine was only used in $17.1 \%$ of the cases. Food related anaphylaxis and other typical triggers of anaphylaxis are age dependent and the risks and triggers change with age. Epinephrine injection should be increased by improving the awareness of physician and medical teams. The study was approved by the Ethics Committee of the Faculty of Medicine of Mashhad University of Medical Sciences (approved number: IR.MUMS.REC.1393.960).

Key words: anaphylaxis; etiology; treatment; survey; allergy; epinephrine; corticosteroid

doi: $10.4103 / 2045-9912.248262$

How to cite this article: Ahanchian H, Behmanesh F, Azad FJ, Ansari E, Khoshkhui M, Farid R, Hassanpur Y, Kouzegaran S. A survey of anaphylaxis etiology and treatment. Med Gas Res. 2018;8(4):129-134.

Funding: The study was supported by Mashhad University of Medical Sciences and Mashhad University of Medical Sciences Office of Research Administration, Iran.

\section{INTRODUCTION}

Anaphylaxis is an acute multi-systemic and potentially fatal reaction, resulting from the rapid release of inflammatory mediators by mast cells and basophils, and occurs when a susceptible person is exposed to a certain agent. ${ }^{1}$ With the discovery of immunoglobulin E (IgE), it became apparent that anaphylactic reactions were in many instances mediated by this antibody. However, not all episodes could be attributed to an IgE-mediated mechanism. ${ }^{2,3}$ Although any substance has the potential to cause anaphylaxis, the most common causes of IgE-mediated anaphylaxis are: foods, particularly, peanuts, tree nuts, shellfish and fish, cow's milk, eggs and wheat; medications (most commonly penicillin), and natural rubber latex. ${ }^{4}$ Exercise, aspirin, non-steroidal anti-inflammatory drugs, opiates, and radiocontrast agents can also cause anaphylaxis, but anaphylactic reactions to these agents often result from non-IgE-mediated mechanisms. ${ }^{5}$ In other cases, the cause of anaphylactic reactions is unknown (idiopathic anaphylaxis). ${ }^{6}$

In children, anaphylaxis is most often caused by foods, while venom- and drug-induced anaphylaxis is more common in adults. The clinical manifestations of anaphylaxis and anaphylactoid reactions are the same for children and adults. The signs and symptoms vary and can range from mild skin findings to a fatal reaction. Ninety percent of patients present with cutaneous symptoms, including urticaria, angioedema, flushing, and warmth; the absence of dermal symptoms does not exclude the diagnosis of anaphylaxis. ${ }^{7}$ Other affected organ systems include the respiratory tract (rhinorrhea, oropharyngeal edema, laryngeal edema, hoarseness, stridor, wheezing, dyspnea, and asphyxiation), cardiovascular system (tachycardia, hypotension, shock, syncope, and arrhythmias), gastrointestinal tract (nausea, abdominal pain, crampy diarrhea, and vomiting), and neurologic system (syncope, seizure, dizziness, and a sense of impending doom) ${ }^{8-12}$ The severity of an anaphylactic reaction is often proportionate to the speed of symptom onset. In anaphylaxis, some of the most common diagnostic dilemmas involve acute asthma, syncope, and anxiety/panic attacks. ${ }^{13,14}$

The acute treatment of anaphylaxis begins with rapid assessment of the airway, breathing and circulation. Epinephrine is the drug of choice for anaphylaxis and should be given immediately to any patient with a suspected anaphylactic episode followed by intramuscular or intravenous $\mathrm{H}_{1}$ and $\mathrm{H}_{2}$ antihistamine antagonists, oxygen, intravenous fluids, inhaled $\beta$-agonists, and corticosteroids. ${ }^{15}$ Considering that the most important principle in the prevention and treatment of allergies is avoiding allergens, management of anaphylaxis focuses on identifying and avoidance of known triggers. Moreover, Educating the patient and family members about the signs and symptoms of anaphylaxis and using self-administered epinephrine early, result in better outcomes. ${ }^{16}$

As many factors including the cultural habits, diet and education affect the anaphylaxis causes and treatment, the aim of this study was to evaluate the etiology, common clinical manifestation and treatment strategies of anaphylaxis in Mashhad, northeast of Iran. 


\section{Subjects AND Methods Subjects}

In this observational cross-sectional study, we reviewed records of all patients $(n=70)$ with a history of anaphylactic reaction who were referred to University Allergy Clinics of Mashhad, Iran between 2006 and 2016.

Inclusion criteria: All patients with a history of anaphylactic reaction who referred to allergy clinics of Mashhad Medical University.

Exclusion criteria: All patients with a history of seizure, cardiovascular disease or panic attack.

After taking informed consent from the participants (adult patients and children parents), a detailed clinical history regarding the main triggers of anaphylactic reaction from the patient's point of view including the agents patient encountered before the reaction (e.g., foods, medications or insect bites/ stings), as well as the patient's activities preceding the event (e.g., exercise, sexual activity) were taken. Number of anaphylactic attacks, clinical manifestations, and treatments received by the patients at the time of attacks, patient's demographic data, age and sex of the patients, presence of atopy and family history of anaphylaxis were recorded in checklists.

The diagnostic work up for detection of specific IgE included the following: In the first step, radioallergosorbant test was done for 30 common food allergens (milk, egg, nuts, soy, fruits, etc.) or common venom allergens based on history. Skin prick test (SPT) for common food allergens or venoms was performed in patients whose clinical history and radioallergosorbant test (RAST) could not determine the etiology of attacks. SPT performed in hospital to be able to treat possible anaphylactic reaction. SPT was done for some common food allergens such as milk, egg, nuts, soy, and fruits in addition to the suspected agent based on medical history. However, in other cases such as drug or insect bite, SPT was performed only with the suspected agent. If history, skin prick test and RAST test did not specify the cause of anaphylaxis is classified as "idiopathic". ${ }^{17}$

\section{Skin prick test}

In SPT, the location of each allergen can be marked with a pen or by using a test grid on the forearm to properly identify test results. Tests should be applied to the volar aspect of the forearm, at least $2-3 \mathrm{~cm}$ from the wrist and the antecubital fossae. A drop of each test solution should be placed on the skin in identical order for each subject tested and immediately pricked via a lancet. Positive and negative controls should be measured first. The negative control excludes the presence of dermographism which makes the tests difficult to interpret. The histamine control should be positive to make sure that the test materials are applied correctly and to exclude negative SPT results due to potentially interfering medications taken by the test subject 11 . The resulting wheal and flare reactions of histamine were read at 10 minutes and those of allergens at 20 minutes. The skin test considered positive if the diameter of the wheal was $3 \mathrm{~mm}$ or more. ${ }^{18}$

\section{Radioallergosorbant test}

RAST involves a sandwich method in which specific antigen is attached to a solid-phase paper disc (allergosorbent). The solid-phase disc is incubated with the patient's serum, which contains IgE antibody. Unbound antibody is removed, and radiolabeled anti-IgE antibody added. A gamma scintillation counter measures the amount of radioactivity as a reflection of the amount of specific IgE in the patient's serum. Results are compared with standard reference sera. ${ }^{19}$

\section{Statistical analysis}

Data gathered from answered questionnaires were classified, tabulated and analyzed using IBM SPSS version 23.0 (IBM Co., Armonk, NY, USA) and Excel (Microsoft, Redmond, WA, USA). We used multiple response analysis and cross tabulation to analyze the data and descriptive statistics generated.

\section{Ethical considerations}

Since all patients gave informed consent after being informed of the study and risks and benefits of allergy testing, ethical issues were taken into consideration.

\section{License ethics committee code}

This research was approved by Ethics Committee of the Faculty of Medicine of Mashhad University of Medical Sciences (approved number: IR.MUMS.REC.1393.960).

\section{RESULTS}

\section{Age and gender distribution of patients}

We identified 172 anaphylactic reactions in 70 patients with median age of 15 years (range from 6 months to 48 years). Thirty-nine cases were children under 19 years of age $(\leq 19$ years). Demographics showed a predominance of male gender among cases (39 males, 55.7\%); however, there was a higher proportion of female gender in adults aged 30 to 39 years and a similar distribution of the sexes in adolescence. Participants were categorized based on their age at time of reaction into 6 strata (Figure 1). $24.3 \%$ were preschoolers ( $0-5$ years), $20 \%$ were schoolchildren (6-12 years), 11.4\% were adolescents/ teenagers (13-19 years), 17.1\% were young adults (20-29 years) and $14.3 \%$ aged (30-39 years) in addition to $12.9 \%$ aged (40-49 years) were categorized as middle-aged adults. The highest number of anaphylaxis cases were in preschool age group ( $<6$ years, $0-5)$ followed by schoolchildren and young adults aged 20 to 29 years (Figure 1).

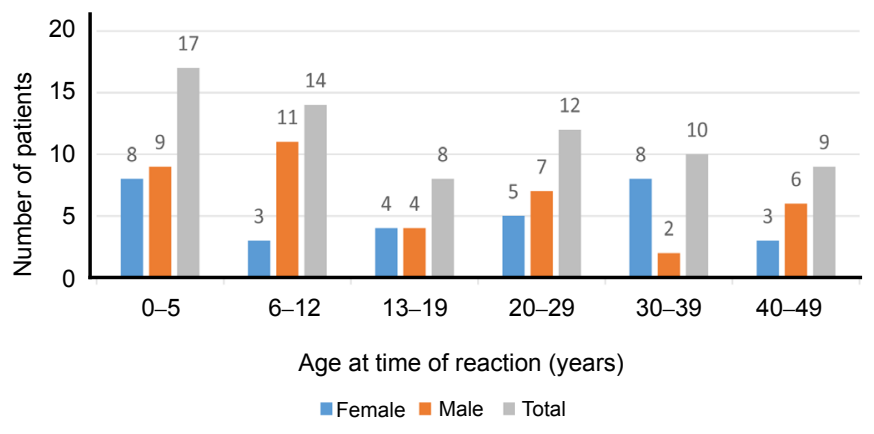

Figure 1: Age and gender distribution of patients. 


\section{Number of attacks per patient}

A total of 172 anaphylactic reactions were recognized. Fortyfour patients had experienced only one episode of anaphylactic reaction whereas 26 got more than one attack. The highest number of anaphylactic attacks (30 attacks) were due to unknown cause (recurrent idiopathic anaphylaxis) in a middleaged female (40 years), followed by 22 attacks due to Wasp bite in a middle aged male farmer ( 45 years). There were also 10 anaphylactic attacks due to exercise ( 8 times) and food (2 times) in a teenage male (17 years) (Table 1). Although atopy was present in two thirds of the patients $(64.3 \%)$, only 2 patients $(2.9 \%)$ had a positive family history of anaphylaxis.

\begin{tabular}{llll}
\hline $\begin{array}{l}\text { Table 1: Number of attacks per patient and total number } \\
\text { of attacks }\end{array}$ & & \\
\hline Anaphylactic attacks & Patients & Percentage & Total attacks \\
\hline 1 & 44 & 62.9 & 44 \\
2 & 9 & 12.9 & 18 \\
3 & 11 & 15.7 & 33 \\
4 & 1 & 1.4 & 4 \\
5 & 1 & 1.4 & 5 \\
6 & 1 & 1.4 & 6 \\
10 & 1 & 1.4 & 10 \\
22 & 1 & 1.4 & 22 \\
30 & 1 & 1.4 & 30 \\
\hline Total & 70 & 100 & 172 \\
\hline
\end{tabular}

\section{Distribution of anaphylaxis triggers}

Overall, food was found to be the most common cause of anaphylaxis $(61.4 \%)$, followed by drugs $(15.7 \%)$ and stinging of hymenoptera in $8.6 \%$ of patients. In 4 cases $(5.7 \%)$ no identifiable cause was found (idiopathic anaphylaxis). Immunotherapy was the cause of anaphylaxis in 3 patients $(4.3 \%)$ and other causes were noted in 4 patients $(5.7 \%$ ) (Figure 2). Other causes include: Food dependent exercise induced anaphylaxis due to wheat in 2 adult patients ( 3 attacks each one), exercise induced anaphylaxis (food independent) in a teenager, in whom also a type of shellfish (shrimp) was a trigger of anaphylaxis (total attacks 10), and the last one was due to horse dander in a school aged child ( 2 attacks).

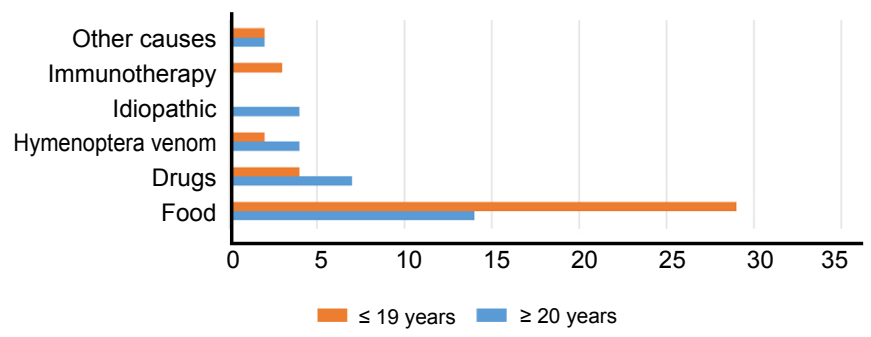

Figure 2: Distribution of anaphylaxis triggers in children and adults.

\section{Main triggering agents of anaphylaxis}

Among foods, nuts especially walnut $(n=9)$ and peanut ( $n=$ 6 ) were the most prevalent allergens triggering anaphylaxis followed by fruits $(n=6)$. Antibiotics $(n=5)$ and local anesthetics $(n=4)$ accounted for $82 \%$ of drug induced anaphylaxis. Other drugs included: a herbal drug named Shekar tighal (Trehala manna) in a 38-year-old female, and in the other case, anaphylaxis occurred in a 33-year-old female using different trademark of acetaminophen probably due to drug excipients. Among insects, 5 cases of insect venom anaphylaxis were due to yellow jacket and 1 was due to wasp. It is worth to be noted that in some cases, specifically in some cases of food induced anaphylaxis more than one food or cause was recognized as triggering agent (Table 2).

Most cases of food related anaphylaxis occurred in children, adolescents and young adults especially in preschool aged children (0-5 years) (Figure 3). Furthermore, in general, Treenuts/Peanuts/Seeds were the predominant triggers of food induced anaphylaxis among cases $(n=23)$, especially in schoolchildren $(n=6)$, adolescents $(n=5)$ and young adults $(n=6)$. However, cow's milk and hen's egg were the main triggers of anaphylaxis in children under 2 years of age. Fruit as the second main cause of food induced anaphylaxis $(n=6)$ was the causative agent in both children and adults and was present in all age groups as triggering agent except for adolescents. Spices (curry and pepper) were the cause of anaphylactic reaction in adulthood (Table 2).

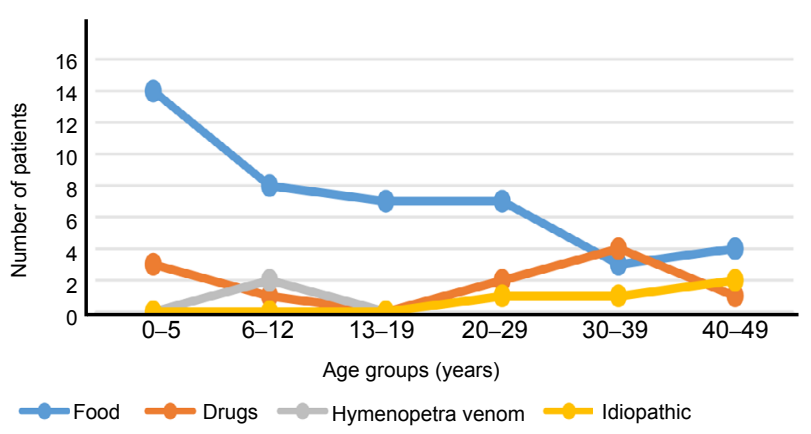

Figure 3: Distribution of four main causes of anaphylaxis in different age groups.

Drugs as the second most important causes of anaphylaxis $(n=11)$ were more reported in adults above 20 years ( 7 cases) compared to children under 19 years ( 4 cases. Stinging of hymenoptera $(n=6)$ as other main causes of anaphylaxis were also more common in adults $(n=4)$ compared to children $(n$ $=2)$. Finally, idiopathic anaphylaxis $(n=4)$ as another main cause of anaphylaxis was only present in adults. Three of four cases of idiopathic anaphylaxis occurred in middle aged female adults (aged 38, 40 and 47 years) and the other one occurred in a young male adult aged 25 (Figures $2 \& 3$ and Table 2 ).

The most frequent clinical features of anaphylaxis during an acute episode were mucocutaneous $(85.7 \%)$ and cardiovascular $(77.1 \%)$ features, followed by respiratory in $71.4 \%$ and gastrointestinal features in $15.7 \%$ of the cases (Figure 4). No fatalities occurred in our series of patients. The most severe reaction was a cardio-pulmonary arrest in a 3-year-old girl due to a type of freshwater fish named Tilapia which resolved after cardiopulmonary resuscitation. The majority of patients diagnosed in this study (77.1\%) appear to have had severe 


\section{Table 2: Main triggering agents of anaphylaxis according to the age of patients}

\begin{tabular}{|c|c|c|c|c|c|c|c|}
\hline & \multicolumn{7}{|c|}{ Age groups (years) } \\
\hline & $0-5$ & 6-12 & $13-19$ & $20-29$ & 30-39 & $40-49$ & 70 \\
\hline Agents & $17(24.3)$ & $14(20.0)$ & $8(11.4)$ & $12(17.1)$ & $10(14.3)$ & $9(12.9)$ & $70(100.0)$ \\
\hline \multicolumn{8}{|l|}{ Foods } \\
\hline \multicolumn{8}{|l|}{ Nuts/Seeds } \\
\hline Hazelnut & - & $1(7.1)$ & - & $1(8.3)$ & - & - & $2(2.9)$ \\
\hline Walnut & $3(17.6)$ & $2(14.3)$ & $1(12.5)$ & $2(16.7)$ & - & $1(11.1)$ & $9(12.9)$ \\
\hline Almond & - & $1(7.1)$ & - & $1(8.3)$ & - & - & $2(2.9)$ \\
\hline Peanut & - & $1(7.1)$ & $3(37.5)$ & $1(8.3)$ & - & $1(11.1)$ & $6(8.6)$ \\
\hline Sunflower seed & $1(5.9)$ & $1(7.1)$ & - & - & - & - & $2(2.9)$ \\
\hline Sesame & - & - & $1(12.5)$ & $1(8.3)$ & - & - & $2(2.9)$ \\
\hline \multicolumn{8}{|l|}{ Fruits } \\
\hline Pomegranate & - & $1(7.1)$ & - & - & $1(10.0)$ & - & $2(2.9)$ \\
\hline Peach & - & $1(7.1)$ & - & - & - & - & $1(1.4)$ \\
\hline Kiwi & - & - & - & - & - & $1(11.1)$ & $1(1.4)$ \\
\hline Strawberry & $1(5.9)$ & - & - & - & - & - & $1(1.4)$ \\
\hline Grape & - & - & - & $1(8.3)$ & - & - & $1(1.4)$ \\
\hline \multicolumn{8}{|l|}{ Fish/Shellfish } \\
\hline Freshwater fish & $2(11.8)$ & - & - & $1(8.3)$ & - & - & $3(4.3)$ \\
\hline Shrimp & - & - & $1(12.5)$ & - & - & - & $1(1.4)$ \\
\hline \multicolumn{8}{|l|}{ Spices } \\
\hline Curry & - & - & - & $1(8.3)$ & $1(10.0)$ & - & $2(2.9)$ \\
\hline Pepper & - & - & - & $1(8.3)$ & - & - & $1(1.4)$ \\
\hline \multicolumn{8}{|l|}{ Other foods } \\
\hline \multicolumn{8}{|l|}{ Grain } \\
\hline Wheat & - & - & - & - & - & $1(11.1)$ & $1(1.4)$ \\
\hline Corn & $1(5.9)$ & - & - & - & - & - & $1(1.4)$ \\
\hline \multicolumn{8}{|l|}{ Hen’s egg } \\
\hline Egg white & $3(17.6)$ & - & - & - & - & - & $3(4.3)$ \\
\hline Egg yolk & $1(5.9)$ & - & - & - & $1(10.0)$ & - & $2(2.9)$ \\
\hline Milk & $1(5.9)$ & - & - & - & - & - & $1(1.4)$ \\
\hline Soy & $1(5.9)$ & - & - & - & - & - & $1(1.4)$ \\
\hline Potato & $1(5.9)$ & $1(7.1)$ & - & - & - & - & $2(2.9)$ \\
\hline \multicolumn{8}{|l|}{ Drugs } \\
\hline \multicolumn{8}{|l|}{ Antibiotics } \\
\hline Penaicillin & $3(17.6)$ & - & - & - & - & - & $3(4.3)$ \\
\hline Ceftriaxone IV & - & - & - & $1(8.3)$ & - & - & $1(1.4)$ \\
\hline Ciprofloxacin & - & - & - & - & $1(10.0)$ & - & $1(1.4)$ \\
\hline \multicolumn{8}{|l|}{ Local anesthetics } \\
\hline Prilocaine & - & - & - & - & - & $1(11.0)$ & $1(1.4)$ \\
\hline
\end{tabular}

Note: Data are expressed as number (percent).

anaphylactic reactions associated with hypotension/shock and cardiovascular involvement, which is much higher than usual 30 percent incidence of cardiovascular involvement in anaphylactic reactions.

Single or associate medications used to treat the acute episodes were systemic corticosteroids in $94.3 \%$ of the patients, antihistamines in $85.7 \%$ and intravenous fluids in $54.3 \%$ and epinephrine in $17.1 \%$ (Figure 5). Despite the fact that the major treatment of anaphylaxis is intramuscular epinephrine, it was only used in a minority of cases (17.1\%) in our study and the majority of patients were treated with corticosteroids and/or antihistamines.

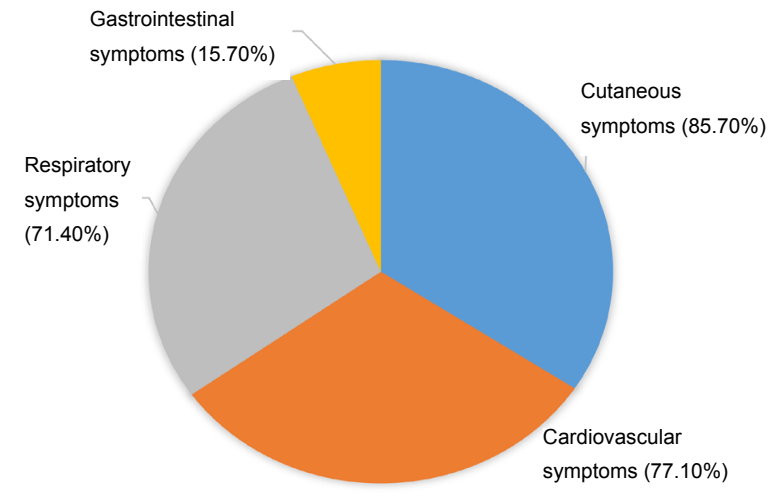

Figure 4: Categorization of organ systems involved. 


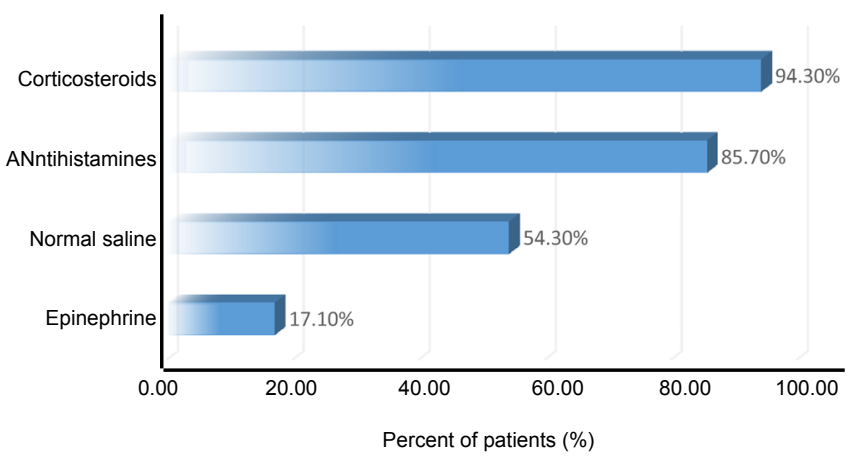

Figure 5: Acute treatment of anaphylaxis at the time of attack.

\section{DISCUSSION}

Considering that the most important principle in the prevention and treatment of allergies is avoiding allergens, management of anaphylaxis focuses on identifying and avoidance of known triggers. Furthermore, a referral to an allergy specialist for a thorough history, diagnostic testing, and recommendations for avoidance is suggested for patients following severe allergic reactions or anaphylaxis. Skin testing and serum IgE-specific testing are available for foods, inhalants, insect venoms, drugs (penicillin), vaccines, and latex. Moreover, educating the patient and family members about the signs and symptoms of anaphylaxis and using self-administered epinephrine early, result in better outcomes. Fatal anaphylaxis has occurred despite timely and appropriate treatment. A MedicAlert bracelet with appropriate information is also recommended. According to recent studies, anaphylaxis incidence is increasing. ${ }^{20-22}$ It is more prevalent especially in the first two decades of life. In the present study, the highest anaphylaxis incidence was observed in preschool age ( $0-5$ years old) and then at school ages (6-12 years old) and young ages (20-29 years old), respectively. In another study in Florida, similar to our study, reported the highest incidence in children below 17 years old. ${ }^{23}$

In the present study, the most common cause of anaphylaxis during childhood (less than 19 years old) was food. Other causes of anaphylaxis incidence in this age group included foods, medicines, hymenoptera venom, other causes, and immunotherapy. In most studies, food is also the most common cause of anaphylaxis in children, but the type of food is different among studies. ${ }^{24}$

In our study, in adults (over 20 years old), the most common causes of anaphylaxis were foods, medicines, hymenoptera venom, idiopathic and miscellaneous causes, respectively. Contrary to the present study, in a retrospective study conducted by Yoon et al. ${ }^{13}$ at Pusan University Hospital in Busan, Korea from January 2009 to June 2014 on adults with anaphylaxis the most common causes of anaphylaxis incidence were medicines, foods, insects' bite, food-independent exercise-induced anaphylaxis, food-dependent exercise-induced anaphylaxis, and idiopathic causes.

According to the existing information, the role of atopy and allergies has been indicated in anaphylaxis incidence. In this study, about two thirds of patients with anaphylaxis had a history of atopy. In the study by Yoon et al., ${ }^{13} 56 \%$ patients had a history of allergic diseases that was similar to our study.

In the current study, food anaphylaxis was mostly due to the consumption of nuts, especially walnut, peanut, as well as with lower incidence by fruit consumption. While eggs and cow milk in children younger than 2 years old was the most common cause of food anaphylaxis. In European and American studies similar to our study, peanut and other nuts are the most common cause of food anaphylaxis, ${ }^{25-29}$ while in a study conducted in Korea the most commonly causes included buckwheat, fish, and eggs. ${ }^{30}$ The difference in anaphylaxis factor probably in these studies is related to the style and habit of food in different regions. However, the common point in all studies was the change in the type of anaphylaxis factor due to age, so that milk was the most common cause at the age of less than 2 years old.

In our study, among medicines, antibiotics and topical anesthetics were the most common cause of medicine anaphylaxis. Medicine anaphylaxis was also higher in adults than in children. Another study conducted in the United States also showed similar results to our study. ${ }^{25}$ In our study, anaphylaxis incidence was caused by insects' bite $(8.6 \%)$ which was mainly related to yellow bees' bite. In other studies, anaphylaxis also varied from $3 \%$ to $10 \%$, which is consistent with our study. ${ }^{13}$

In the present study, the most common manifestations of anaphylaxis were mucocutaneus $(85.7 \%)$, cardiovascular $(77.1 \%)$, respiratory $(71.4 \%)$ and gastric $(15.7 \%)$ manifestations, respectively. Studies conducted in the United States, Europe, South Korea and Portugal have shown similar results. ${ }^{13,25,31,32}$ In adults, hypotension was more common than in children. The reason for this was probably due to more difficult measurement of blood pressure in children, especially those younger than 5 years old. As the age increases in adults, the prevalence of heart disease is also increases. ${ }^{33,34}$

In this study, more acute reactions were treated with corticosteroids and antihistamines, and only less than one quarter of patients $(17.1 \%)$ received epinephrine. It seems that, despite the recommendations of anaphylaxis treatment guidelines based on the role of epinephrine as the main treatment, threequarters of the patients did not receive adequate treatment. Perhaps this problem would be resolved with increasing the medical personnel awareness.

The limitations of our study include lack of laboratory evaluation of anaphylaxis attacks. The advantage of our study is the number of patients, practical information and interesting results for the first time this region.

In summary, foods (nuts) are the most common triggers of anaphylaxis in Mashhad. There is a significant epinephrine undertreatment in anaphylaxis management in this region, which needs educational plans to increase awareness of physicians all medical staffs.

\section{Author contributions}

Designing of the study: HA, FB; data gathering: $\mathrm{YH}$; diagnosis and treatment of the patients: FJA, EA, MK, RF; manuscript writing: SK. Conflicts of interest

There is no conflict of interest.

Financial support

The study was supported by Mashhad University of Medical Sciences and Mashhad University of Medical Sciences Office of Research Administration, Iran.

Institutional review board statement

The study was approved by the Ethics Committee of the Faculty of 
Medicine of Mashhad University of Medical Sciences (approved number: IR.MUMS.REC.1393.960).

Declaration of patient consent

The authors certify that they have obtained patient consent forms. In the form, patients have given their consent for their images and other clinical information to be reported in the journal. The patients understand that their names and initials not be published and due efforts will be made to conceal their identity, but anonymity cannot be guaranteed. Biostatistics statement

The statistical methods of this study were reviewed by the biostatistician of University of Queensland, Brisbane, Australia.

Copyright license agreement

The Copyright License Agreement has been signed by all authors before publication.

Data sharing statement

Individual participant data that underlie the results reported in this article, after deidentification (text, tables, figures, and appendices). Study protocol and informed consent form will be available immediately following publication, without end date. Results will be disseminated through presentations at scientific meetings and/or by publication in a peer-reviewed journal. Anonymized trial data will be available indefinitely at www.figshare.com.

\section{Plagiarism check}

Checked twice by iThenticate.

\section{Peer review}

Externally peer reviewed.

Open access statement

This is an open access journal, and articles are distributed under the terms of the Creative Commons Attribution-NonCommercialShareAlike 4.0 License, which allows others to remix, tweak, and build upon the work non-commercially, as long as appropriate credit is given and the new creations are licensed under the identical terms.

\section{REFERENCES}

1. Nagano C, Ishiguro A, Yotani N, Sakai H, Fujiwara T, Ohya Y. Anaphylaxis and biphasic reaction in a children hospital. Arerugi. 2013;62:163-170.

2. Peterson MR, Coop CA. Long-term omalizumab use in the treatment of exercise-induced anaphylaxis. Allergy Rhinol (Providence). 2017;8:170-172.

3. Nakamura T, Fukaya T, Uto T, et al. Selective depletion of basophils ameliorates immunoglobulin E-mediated anaphylaxis. Biochem Biophys Rep. 2016;9:29-35.

4. Plagova A, Nykamp D. Recognition and management of anaphylaxis in older adults. Consult Pharm. 2017;32:398-405.

5. Sreevastava D, Tarneja V. Anaphylactic reaction: An overview. Med J Armed Forces India. 2003;59:53-56.

6. Nwaru BI, Dhami S, Sheikh A. Idiopathic anaphylaxis. Curr Treat Options Allergy. 2017;4:312-319.

7. Cianferoni A, Khullar K, Saltzman R, et al. Oral food challenge to wheat: a near-fatal anaphylaxis and review of 93 food challenges in children. World Allergy Organ J. 2013;6:14.

8. Agelebe E, Musa TL, Ajayi IA, Oyedeji OA. Anaphylaxis complicated by acute respiratory distress and fatal outcome in a Nigerian family. J Clin Diagn Res. 2017;11:SD01-SD03.

9. Foroutan M, Loloei B, Irvani S, Azargashb E. Accuracy of rapid urease test in diagnosing Helicobacter pylori infection in patients using NSAIDs. Saudi J Gastroenterol. 2010;16:110-112.

10. Darvishi M, Forootan M, Azmodeh O, Forootan M, Matinfar H. Studying the frequency of helicobacter pylori infection among women suffering from polycystic ovary resorting to hospital infertility center. Biomed Pharmacol J. 2017;10:75-80.

11. Johari Moghadam A, Azizinejad S. Study of high sensitive c-reactive protein (HS-CRP) after cardiac rehabilitation program in patients undergoing isolated CABG. Int J Biomed Sci. 2016;12:143148.

12. Mansueto P, Montalto G, Pacor ML, et al. Food allergy in gastroenterologic diseases: Review of literature. World J Gastroenterol. 2006; 12:7744-7752.

13. Yoon L, Kim BR, Lee JY, et al. Clinical features of anaphylaxis according to age in a single university hospital in Korea. Asian Pac J Allergy Immunol. 2017;35:96-101.

14. Simons FE, Ardusso LR, Bilò MB, et al. World allergy organization guidelines for the assessment and management of anaphylaxis. World Allergy Organ J. 2011;4:13-37.
15. Alvarez-Perea A, Tanno LK, Baeza ML. How to manage anaphylaxis in primary care. Clin Transl Allergy. 2017;7:45.

16. Sicherer SH, Sampson HA. Food allergy: a review and update on epidemiology, pathogenesis, diagnosis, prevention, and management. J Allergy Clin Immunol. 2018;141:41-58.

17. Barzegar S, Rosita A, Pourpak Z, et al. Common causes of anaphylaxis in children: the first report of anaphylaxis registry in Iran. World Allergy Organ J. 2010;3:9-13.

18. Hong SD, Ryu G, Seo MY, et al. Optimal cutoff values of allergenspecific immunoglobulin $\mathrm{E}$ to house dust mites and animal dander based on skin-prick test results: Analysis in 16,209 patients with allergic rhinitis. Am J Rhinol Allergy. 2018;32:23-26.

19. Kianifar HR, Pourreza A, Azad FJ, Yousefzadeh H, Masomi F. Sensitivity comparison of the skin prick test and serum and fecal radio allergosorbent test (RAST) in diagnosis of food allergy in children. Rep Biochem Mol Biol. 2016;4:98-103.

20. Decker WW, Campbell RL, Manivannan V, et al. The etiology and incidence of anaphylaxis in Rochester, Minnesota: a report from the Rochester Epidemiology Project. J Allergy Clin Immunol. 2008;122:1161-1165.

21. Sheikh A, Alves B. Hospital admissions for acute anaphylaxis: time trend study. BMJ. 2000;320:1441.

22. Turner PJ, Jerschow E, Umasunthar T, et al. Fatal anaphylaxis: mortality rate and risk factors. J Allergy Clin Immunol Pract. 2017;5:1169-1178.

23. Harduar-Morano L, Simon MR, Watkins S, Blackmore C. A population-based epidemiologic study of emergency department visits for anaphylaxis in Florida. J Allergy Clin Immunol. 2011;128:594600 .

24. Huang F, Chawla K, Järvinen KM, Nowak-Węgrzyn A. Anaphylaxis in a New York City pediatric emergency department: triggers, treatments, and outcomes. J Allergy Clin Immunol. 2012;129:162168.

25. Altman AM, Camargo CA, Simons FE, et al. Anaphylaxis in America: a national physician survey. J Allergy Clin Immunol. 2015;135:830-833.

26. Chipps BE. Update in pediatric anaphylaxis: a systematic review. Clin Pediatr (Phila). 2013;52:451-461.

27. Park HM, Noh JC, Park JH, et al. Clinical features of patients with anaphylaxis at a single hospital. Pediatr Allergy Respir Dis. 2012;22:232-238.

28. Simons FE, Sampson HA. Anaphylaxis: unique aspects of clinical diagnosis and management in infants (birth to age 2 years). $\mathrm{J} \mathrm{Al}$ lergy Clin Immunol. 2015;135:1125-1131.

29. Worm M, Eckermann O, Dölle S, et al. Triggers and treatment of anaphylaxis: an analysis of 4000 cases from Germany, Austria and Switzerland. Dtsch Arztebl Int. 2014;111:367-375.

30. Lee SY, Kim KW, Lee HH, et al. Incidence and clinical characteristics of pediatric emergency department visits of children with severe food allergy. J Asthma Allergy Clin Immunol. 2012;32:169175 .

31. Mäkinen-Kiljunen S, Haahtela T. Eight years of severe allergic reactions in Finland: a register-based report. World Allergy Organ J. 2008;1:184-189.

32. Thong B, Cheng Y, Leong K, Tang C, Chng H. Anaphylaxis in adults referred to a clinical immunology/allergy centre in Singapore. Singapore Med J. 2005;46:529-534.

33. Wolk MJ, Bailey SR, Doherty JU, et al. ACCF/AHA/ASE/ASNC/ HFSA/HRS/SCAI/SCCT/SCMR/STS 2013 multimodality appropriate use criteria for the detection and risk assessment of stable ischemic heart disease: a report of the American College of Cardiology Foundation Appropriate Use Criteria Task Force, American Heart Association, American Society of Echocardiography, American Society of Nuclear Cardiology, Heart Failure Society of America, Heart Rhythm Society, Society for Cardiovascular Angiography and Interventions, Society of Cardiovascular Computed Tomography, Society for Cardiovascular Magnetic Resonance, and Society of Thoracic Surgeons. J Am Coll Cardiol. 2014;63:380406.

34. Neaton JD, Wentworth D. Serum cholesterol, blood pressure, cigarette smoking, and death from coronary heart disease overall findings and differences by age for 316099 white men. Arch Intern Med. 1992;152:56-64.

\section{Received: 2018-09-15}

Accepted: 2018-11-02 